\title{
The Role of Endovascular Stents in an Experimental Model of Traumatic Arterial Occlusion - the Temporary Endo-Shunt
}

\author{
Viktor A Reva MD PhD', Marta J Madurska MD², Igor M Samokhvalov MD PhD', \\ Alexey V Denisov MD PhD ${ }^{1}$, Sergey Yu Telickiy MD PhD ${ }^{1}$, \\ Alexey B Seleznev MD PhD', Igor S Zheleznyak MD PhD³, \\ Valery $\mathbf{N}$ Adamenko $\mathrm{MD}^{1}$ and Kenji Inaba $\mathrm{MD}^{4}$ \\ 'Department of War Surgery, Kirov Military Medical Academy, Saint-Petersburg, Russian Federation \\ ${ }^{2}$ R. Adams Cowley Shock Trauma Center, University of Maryland Medical System, Baltimore, USA \\ ${ }^{3}$ Department of Radiology, Kirov Military Medical Academy, Saint-Petersburg, Russian Federation \\ ${ }^{4}$ Department of Surgery, Division of Acute Care Surgery and Surgical Critical Care, Los Angeles County and \\ University of Southern California Medical Center, Los Angeles, USA
}

\begin{abstract}
Background: The aim of this study was to evaluate patency following the deployment of a bare-metal stent (BMS) or covered stent (CS) across a traumatic occlusive peripheral arterial lesion and to estimate the feasibility of urgent stent placement for temporary endovascular shunting.

Methods: Fifteen sheep (25-45 kg) underwent laparoscopic creation of a left external iliac artery (EIA) thrombosis by means of repeated clamping. Sixty minutes after achievement of thrombotic occlusion, animals were randomized into one of three groups: no-treatment (control group), a BMS group, or CS group. Animals were followed up for 30 days with no anticoagulants or anti-platelet drugs administered postoperatively. Doppler ultrasound, computed tomography angiography (CTA), and digital subtraction angiography (DSA) were used to evaluate EIA patency.

Results: Stent implantation resulted in the restoration of in-line flow through the EIA in all cases. The peak systolic velocity (PSV) in the injured limb increased from $10(0-16)$ to $31(28-37) \mathrm{cm} / \mathrm{s}$ in the BMS group $(p<0.0001)$ and from $15(7-18)$ to $24(21-29) \mathrm{cm} / \mathrm{s}$ in the CS group $(p=0.043)$ immediately after stent deployment (both $p=0.001 \mathrm{com}$ pared with the control group). There was no difference in the PSV between the groups at post-injury day 3 , and thereafter. Day-14 CTA and day-30 DSA demonstrated only one patent stent in each study group.

Conclusions: Urgent stent (BMS or CS) implantation can restore arterial patency of a traumatic occlusion for a short period of time and serve as a temporary endovascular shunt. Distal embolization can complicate this procedure and worsen long-term patency.
\end{abstract}

Keywords: Vascular Trauma; Occlusion; Covered Stent; Bare Metal Stent; Recanalization; Endovascular Trauma Management

\section{Corresponding author:}

Viktor A. Reva MD PhD, Department of War Surgery, Kirov Military Medical Academy, 6, Lebedeva str. Saint-Petersburg, Russian Federation 194044.

Email:vreva@mail.ru

(C) 2020 CC BY 4.0 - in cooperation with Depts. of Cardiothoracic/ Vascular Surgery, General Surgery and Anesthesia, Örebro University Hospital and Örebro University, Sweden

\section{INTRODUCTION}

Blunt arterial injury is often associated with significant morbidity. This is multifactorial due to the extent of vascular injury, resulting ischemia, and surrounding tissue damage $[1,2]$. The magnitude of the problem is compounded if the patient has sustained other life-threatening injuries, which take precedence over extremity reperfusion [2]. Peripheral occlusive vascular injury, if indicated, typically undergoes surgical exploration and control of the injured 
segment. This is followed by either formal vascular reconstruction or temporary shunting depending on the patient's condition and status of the injured extremity [3]. However, both of these approaches can be time consuming, and incur blood loss and trauma [4].

Endovascular techniques are becoming increasingly important in trauma [4-7]. While stent-grafting has become common place in difficult anatomical locations such as the aorta, subclavian artery, and iliac artery, the experience in peripheral lesions is limited $[5,7]$. Currently, there are limited data on the immediate endovascular stenting of the occluded arterial segment $[8,9]$.

While anticoagulation is crucial in endovascular surgery, it is often contraindicated in trauma. Most studies examining stent-grafting in trauma report the use of an anti-platelet agent postoperatively [10-13].

We hypothesized that even in a setting where systemic anticoagulation is not recommended, self-expandable stent implantation can be used for temporary endovascular shunting of an injured major extremity artery. The aim of this study was to evaluate short-term arterial patency following the deployment of a bare metal (BMS) or covered stent (CS) across a traumatic occlusive arterial lesion in the absence of postoperative anticoagulation or anti-platelet drugs.

\section{METHODS}

\section{Overview}

Female non-pregnant sheep, weighing between 25 and $45 \mathrm{~kg}$, were utilized in this study, which was approved by the local institutional ethics committee. The study protocol consisted of four phases: preparation, injury, intervention (BMS $(n=5)$, CS $(n=5)$; or control $(n=5))$, and follow-up (Figure 1).

\section{Animal Preparation}

Intramuscular (IM) injection of $10 \mathrm{mg} / \mathrm{kg}$ tiletamine and zolazepam (Zoletil ${ }^{\oplus}$; Virbac, Carros, France) was performed for induction of general anesthesia. Isoflurane at $1-2 \%$ concentration was used to maintain anesthesia.

A $5-\mathrm{Fr} 11-\mathrm{cm}$ sheath was percutaneously placed into the left external jugular vein for drug and fluid delivery. An 11-Fr 11-cm sheath (Cordis Endovascular, USA) was percutaneously placed in the left carotid artery, and fluoroscopy was used to confirm the tip placement in the brachiocephalic trunk. All sheaths and catheters were flushed with heparinized saline. A mobile fluoroscopy unit (C-arm SM-20HF, Listem Corporation, Republic of Korea) was used for capturing fluoroscopy images. The technique for catheterization of extremity arteries in sheep via the carotid artery, as well as the angiographic survey of the ovine pelvis and hind extremity, are described elsewhere [14]. A non-hydrophilic 0.035"

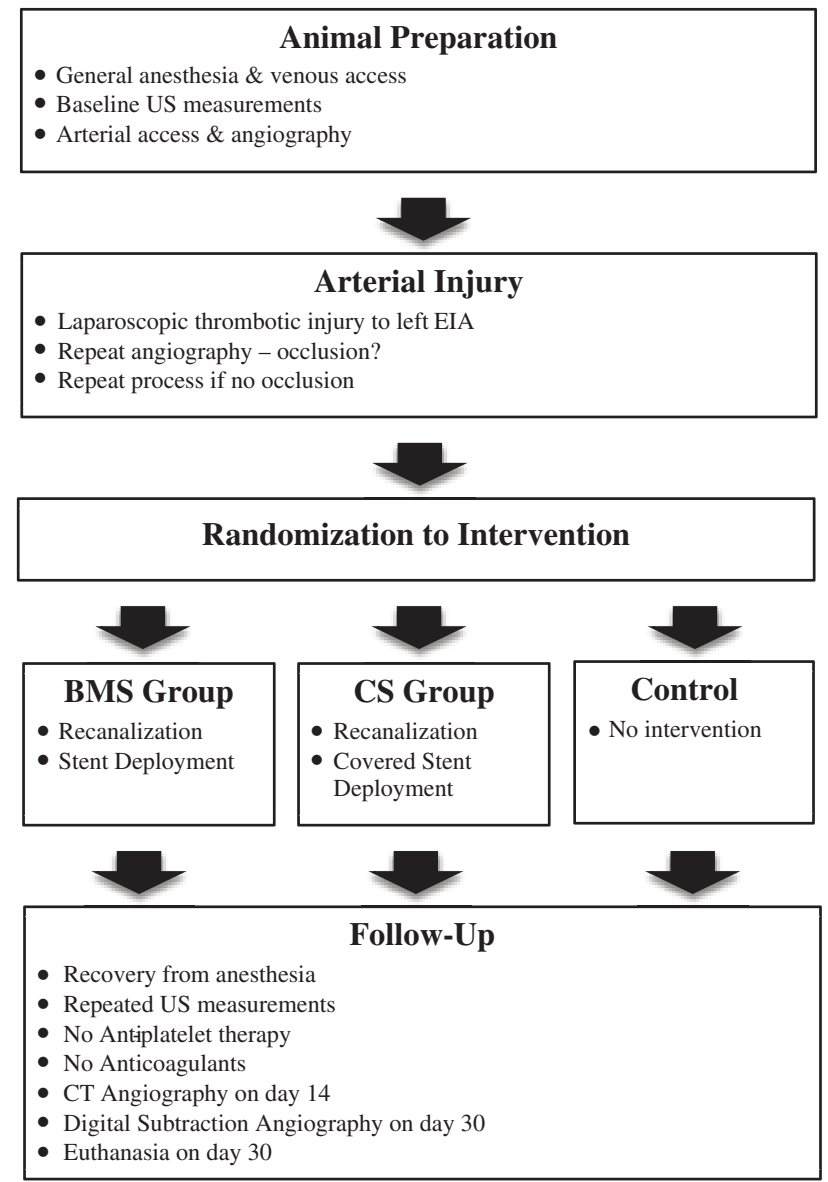

Figure 1 Experimental protocol. BMS: bare metal stent, CS: covered stent, CT: computed tomography, EIA: external iliac artery, US: ultrasound.

Emerald 260-cm wire (Cordis Endovascular, USA) was left in the aorta following angiography.

\section{Laparoscopic Thrombotic Arterial Injury}

A laparoscopic technique was used to develop a traumatic occlusive lesion of the left external iliac artery (EIA; Figure 2). A 10-mm laparoscopic port was placed into the peritoneal cavity, by insertion immediately caudal to the xiphoid process using a Hassan technique. The abdominal cavity was insufflated with carbon dioxide to maintain pneumoperitoneum $(15 \mathrm{~mm} \mathrm{Hg})$. A 5 -mm port was placed just medial to each iliac crest and one additional $10-\mathrm{mm}$ port was placed between the former two parts, immediately left of the midline. The animal was placed in the Trendelenburg position on the right side.

The left EIA was then exposed laparoscopically using diathermy and a dissector. In order to isolate a $3-\mathrm{cm}$ segment of the EIA, the origin of the deep circumflex iliac artery was ligated with 1 or 2 ligaclips via a separate $10-\mathrm{mm}$ trocar. A standardized arterial lesion was then created: a $3-\mathrm{cm}$ section of the artery was controlled, and then 

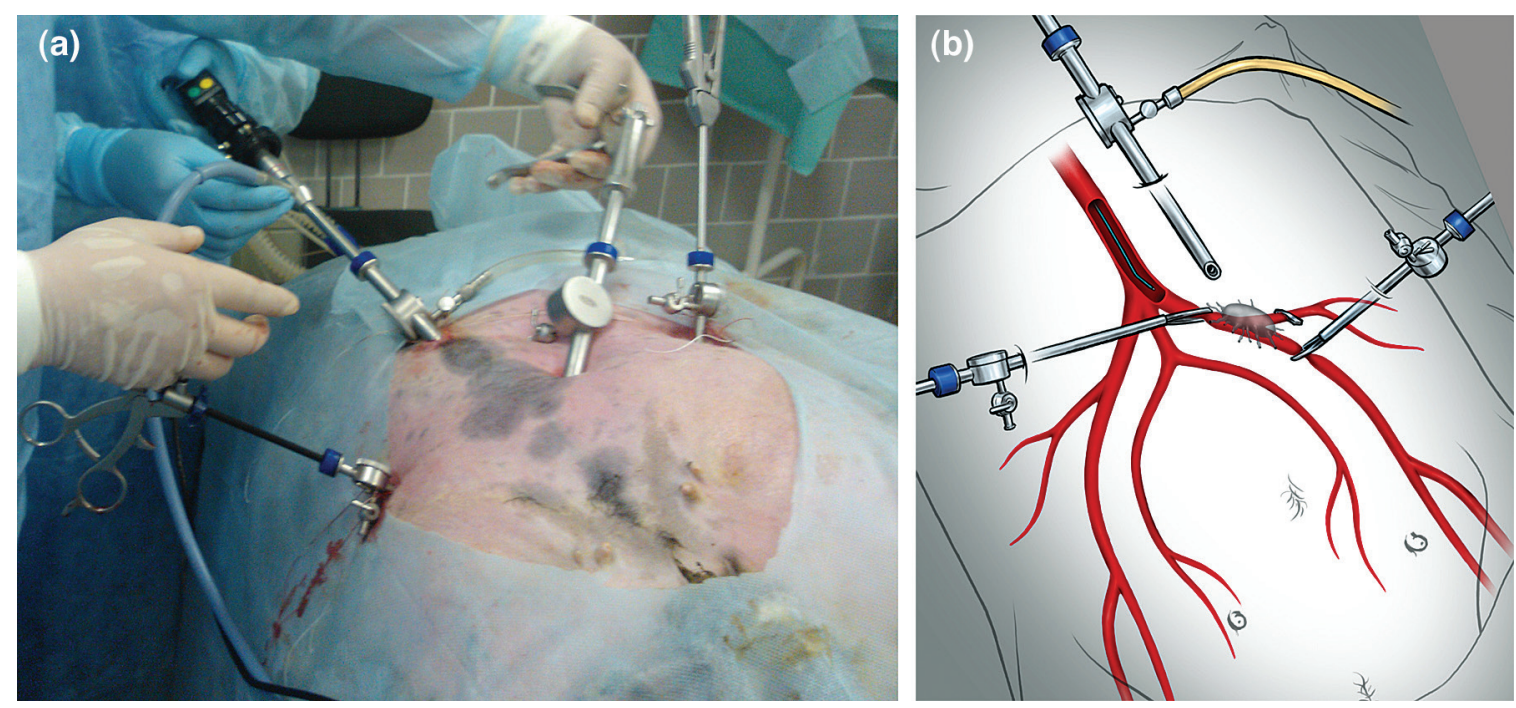

Figure 2 A view of the operative laparoscopic procedure of making occlusive external iliac artery injury. Laparoscopic view. (a) Optimal ports and instruments places are seen. Lower part of the sheep's body is at the bottom. Operative view. (b) A main stage of the laparoscopic procedure is shown. The iliofemoral lymphatic node is located exactly above a targeted zone of the external iliac artery.

clamped repeatedly $(10 \times)$ using grasping forceps and a needle holder in order to induce thrombosis while the forceps were left in place for $30 \mathrm{~min}$. After clamp removal the left EIA underwent repeat angiography in order to confirm thrombosis resulting in full occlusion. If any flow through the injured segment remained, the forceps were applied again for an additional 30 min followed by completion angiography until full occlusion was confirmed. After the completion of the injury, a set of ultrasound (US) measurements was taken at both femoral arteries as post-injury values using the linear transducer $(10-5 \mathrm{MHz})$ of a MicroMaxx ${ }^{\circledR}$ Ultrasound System (Sonosite Inc., USA). At the end of the injury phase, the abdomen was desufflated, all laparoscopic ports were removed, and incisions were closed with interrupted sutures.

\section{Intervention}

Then, 60 min after thrombotic arterial occlusion was achieved, animals in the study groups underwent an endovascular procedure. An indwelling non-hydrophilic wire was exchanged for a 0.035 " hydrophilic guidewire (MIT, Moscow, Russia) which was then used to recanalize the occlusive thrombotic lesion. Once passed through the thrombus, the tip of the wire was left in the distal superficial femoral artery (SFA).

After systemic administration of 2,500 units of Heparin, in the stent groups, a $7 \times 50 \mathrm{~mm}$ nitinol self-expandable or CS (both MIT, Moscow, Russia) overlapping the $3-\mathrm{cm}$ zone of arterial injury was implanted. Neither pre- nor post-implantation dilatation was performed to avoid distal embolization. When post-implantation distal thrombi were identified with angiography, no attempts at thrombus removal were undertaken. After completion angiography, catheters, wires, and sheath were removed and the defect in the carotid artery was sutured with 5-0 polypropylene (Prolene ${ }^{\circledR}$ ).

In the control group, no stenting was performed following injury and the access sheaths were removed 60 min after the EIA thrombosis was obtained.

\section{Follow-up}

Sixty minutes after the intervention phase, post-operative US measurements were taken. The sheep were then extubated and returned into the vivarium with free access to food and water. All animals received $1.0 \mathrm{~g}$ of Cefazoline IM twice a day for 5 days post procedure. No anticoagulants or anti-platelet drugs were administered in the post-operative period.

Hind limb function was evaluated daily by a veterinarian and a surgeon for the first 2 weeks using the modified Tarlov scale (from 0 - unable to sit, paralyzed limb, to 4 - fully ambulatory).

US measurements were performed on post-injury day $1,3,7,14$, and 30 . On post-injury day 14 , the animals underwent computed tomography angiography (CTA). Toshiba Aquilion 16 Slice Scanner was used for CT and $3 \mathrm{ml} / \mathrm{kg}$ of iodine contrast iopromide $370 \mathrm{mg} \mathrm{I} / \mathrm{ml}$ (Ultravist $^{\circledR}$, Bayer, Australia) was automatically injected intra-arterially.

On post-injury day 30, animals were placed under general anesthesia, underwent control angiography (Ziehm RFD C-arm, Germany) via a carotid access to estimate the patency of the iliac, femoral arteries and run-off vessels, followed by euthanasia with an intravenous injection of $100 \mathrm{mEq}$ of potassium chloride. 
Table 1 A comparison of baseline and operative characteristics between groups.

\begin{tabular}{|c|c|c|c|c|}
\hline Parameter & Bare Metal Stent Group & Covered Stent Group & Control Group & $P$ \\
\hline$n$ & 5 & 5 & 5 & \\
\hline Male & $5(100 \%)$ & $5(100 \%)$ & $3(60 \%)$ & 0.099 \\
\hline Weight, kg & $41.0(28.5-43.6)$ & $42.5(31.0-44.0)$ & $25.3(23.2-41.6)$ & 0.156 \\
\hline \multicolumn{5}{|l|}{ Physiology } \\
\hline Heart rate, beats/min & $95(84-120)$ & $110(97-119)$ & $80(72-92)$ & 0.072 \\
\hline Systemic SBP, mm Hg & $120(103-133)$ & $132(113-159)$ & $109(98-123)$ & 0.236 \\
\hline \multicolumn{5}{|l|}{ Thrombosis } \\
\hline Time to creation of injury, min & $60(58-98)$ & $60(43-85)$ & $55(48-60)$ & 0.283 \\
\hline \multicolumn{5}{|l|}{ Operative } \\
\hline Anesthesia time (including laparoscopy), min & $255(230-352)$ & $320(193-345)$ & $200(170-250)$ & 0.204 \\
\hline Time for endovascular procedure, min & $25(23-45)$ & $40(35-53)$ & - & 0.168 \\
\hline Volume of contrast medium, ml & $70(58-70)$ & $80(55-90)$ & $60(40-65)$ & 0.191 \\
\hline \multicolumn{5}{|l|}{ Arterial data } \\
\hline Diameter of EIA, mm & $6.0(6.0-7.0)$ & $7.0(6.8-7.3)$ & $6.0(5.5-6.5)$ & 0.053 \\
\hline
\end{tabular}

Values are median (IQR). EIA: external iliac artery; SBP: systolic blood pressure.

Animals with clinical signs of severe illness (coenurosis cerebralis, diarrhea) or in distress due to extremity dysfunction, were euthanized before the end of the study (EOS), as determined by a veterinarian. A complete autopsy was performed after the completion angiography to examine for any pathology, laparoscopic, or vascular access-related complications.

\section{Study Endpoints and Statistical Analysis}

The primary endpoint of this study was stent patency. Early patency was assessed by quantitative and qualitative Doppler waveform analysis. Blood peak systolic velocity (PSV) in $\mathrm{cm} / \mathrm{s}$, and pulsatility index (PI) comparing the left (injured) and right (control) sides at the following time points: pre- and post-injury, post-operative, at day 1,3,7,14, and 30, were measured. Doppler waveform type in the SFA was also utilized to assess proximal patency.

After animal recovery, CTA and digital subtraction angiography (DSA) allowed evaluation of the run-off vessels for evidence of occluding distal emboli.

Secondary endpoints consisted of laboratory indices of hypocoagulation, reperfusion injury, contrast-induced acute kidney injury (AKI), complications relating to the procedure and/or arterial access, functional gait outcomes, and early euthanasia due to limb-related or systemic complications.

Data were analyzed using IBM SPSS Statistics v21.0 (IBM, Chicago, IL). Variables were evaluated for normal distribution, and nonparametric data were reported as medians with interquartile ranges. Differences in baseline and interventional characteristics between the three groups were tested using the chi-square test for categorical data and the Kruskal-Wallis test for continuous data. Paired continuous data were assessed with the Wilcoxon matched pairs signed rank test. Baseline US parameters were tested using Mann-Whitney
$U$ test, while using repeated US indices - a one-way repeated measure analysis of variance with the Bonferroni correction. Results were considered significant when $p \leq 0.01$.

\section{Ethical Approval}

Ethical approval was given by the local institutional ethics committee.

\section{RESULTS}

\section{Baseline Characteristics, Arterial Injury, and Intervention}

In total, $N=15$ animals had undergone trauma-induced EIA occlusion before being randomly allocated into either the BMS $(n=5)$, CS $(n=5)$, or control $(n=5)$ group. Baseline characteristics and laboratory values were similar amongst the study groups (Table 1 and 2).

Successful occlusion was achieved in all animals as confirmed by angiography and US (Figure 3). Both intervention groups demonstrated a significant reduction in PSV and PI in the injured limbs $(p<0.001)$. While in the uninjured limbs there was no change in the PSV, there was a significant drop in the PI in the BMS group (from $2.86(2.62-4.35)$ to 2.72 (2.54-3.21; $p=0.005))$. The median time to occlusion was $60 \mathrm{~min}$ in the BMS and CS groups, and $55 \mathrm{~min}$ in the control group ( $p=0.283$; Table 1 ).

Successful stent deployment across the vascular injury was achieved in all animals. No animals in either study group showed $>20 \%$ luminal narrowing on completion angiography and no signs of protruding thrombi were seen during stent deployment. An immediate significant increase in PSV and PI for both study groups $(p<0.001)$ was noted following stent deployment (Figure 3). 


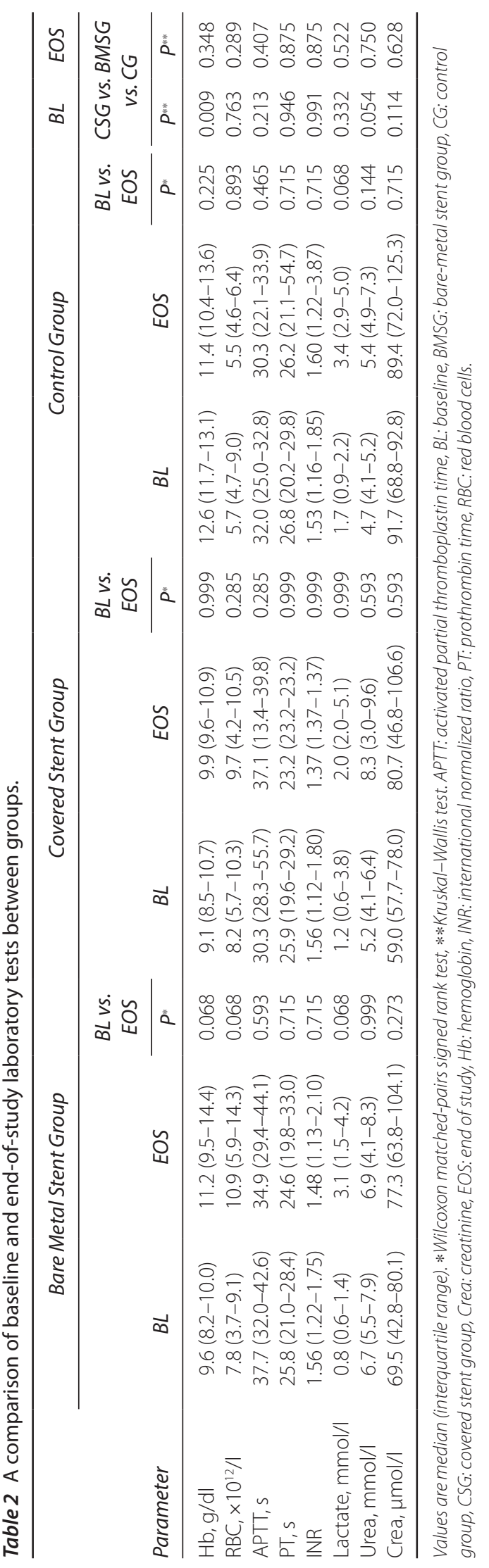

\section{Primary Outcome: Patency}

In addition to stent patency, completion angiography also demonstrated a degree of distal embolization in all animals who had undergone stenting, regardless of the stent type. Occluding fresh thrombi could be found in the profunda femoris, popliteal, and tibial arteries. No fresh thrombi protruding through the cells of the BMSs were seen. In the stent group, $60 \mathrm{~min}$ following stenting a diminished, monophasic arterial flow was recorded in $2 / 5$ and $1 / 5$ animals (BMS and CS groups respectively). In the control group all EIAs were demonstrated to remain occluded 60 min following the injury $(p=0.031)$. There was no significant difference in US measurements between the stent groups in the post-operative time points. Figure 3 demonstrates gradual convergence of PSV and PI in the left (injured) leg between groups. On post-injury day 1 , there was a significant difference in PSV between groups $(p<0.001)$, although the Doppler waveforms demonstrated stent patency in $2 / 5$ and $1 / 5$ animals in the BMS and CS groups, respectively. These results remained unchanged on day 3 post-operation.

On post-injury day 14, CTA showed only one EIA patent in both the BMS and CS groups, with persisting occlusion in the control group. Day 30 DSA confirmed the same arterial patency rate (Table 3 and Figure 4) and demonstrated no difference in collateral development between the stent and control groups. According to CTA and DSA, the blood flow and morphology of distal thrombi were identical between day 14 and day 30 in all groups. In case of stent re-thrombosis, a zone of occlusion was localized and extended only $1-2 \mathrm{~cm}$ in the proximal and distal direction, but not extending to the femoral artery bifurcation. Below-the-knee popliteal or proximal tibial artery occlusion by distal emboli was seen on control angiograms. Non-occluding thrombi were seen in the proximal profunda femoris artery. Run-off vessels were filled by collaterals and seen on angiography.

\section{Secondary Outcomes: Laboratory Indices, Limb Function and Morbidity}

Laboratory baseline and EOS indices presented in Table 2 demonstrate no differences between and within groups. No signs of coagulopathy or AKI were seen.

However, post-operative lactate level was significantly higher in the control group compared with the stent groups ( $p=0.002$, Figure 5$)$. This correlated with early limb dysfunction in the control group as demonstrated by a lower Tarlov gait score $(p>0.05$; Table 3$)$. Ischemic time in this group was much longer than the stent groups where flow was restored 2 hours after thrombosis occurred. Gradual functional improvement was demonstrated in animals in all of the groups, where most animals achieved a Tarlov score of 4 at 7 days, and all animals scored 4 at 14 days. 

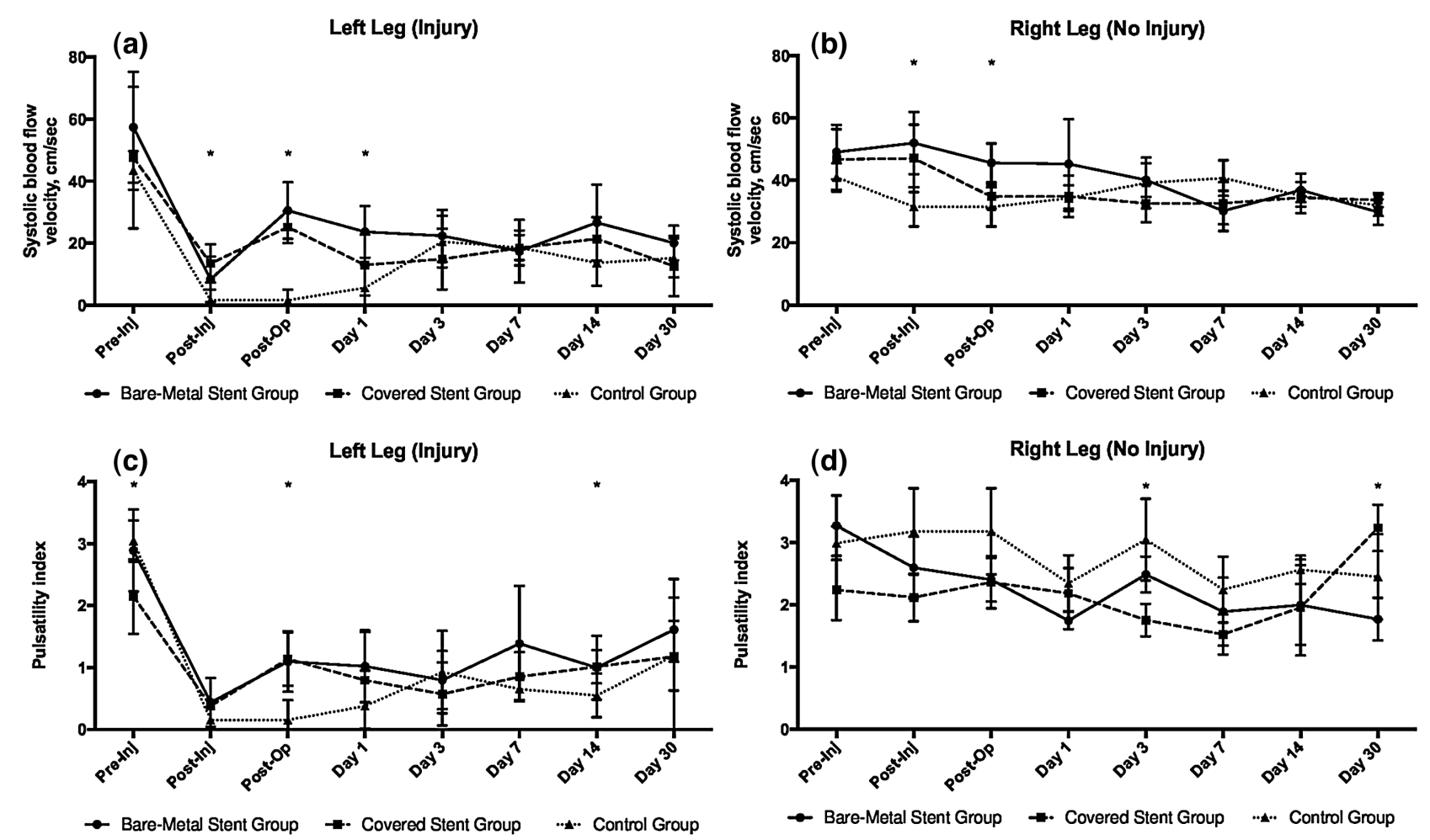

Figure 3 Ultrasonographic measurements of blood flow. (a, b) Systolic blood flow velocity. (c, d) Pulsatility index. Asterisks show significant differences between groups. Data are presented as mean with standard deviation. Pre-inj: pre-injury, Post-inj: post-injury.

Table 3 A comparison of follow-up characteristics between groups.

\begin{tabular}{lcccc}
\hline Parameter & Bare Metal Stent Group & Covered Stent Group & Control Group & $P$ \\
\hline$n$ & 5 & 5 & 5 & \\
Tarlov gait score & & & & \\
$\quad$ Day 1 & $3.0(2.5-3.5)$ & $4.0(2.0-4.0)$ & $2.0(2.0-3.0)$ & 0.121 \\
Day 3 & $4.0(3.0-4.0)$ & $4.0(2.5-4.0)$ & $3.0(2.5-3.5)$ & 0.295 \\
Day 7 & $4.0(3.5-4.0)$ & $4.0(4.0-4.0)$ & $4.0(2.5-4.0)$ & 0.549 \\
Day 14 & $4.0(4.0-4.0)$ & $4.0(4.0-4.0)$ & $4.0(4.0-4.0)$ & 1.000 \\
EOS patency (\%) & $1 / 5(20)$ & $1 / 5(20)$ & $0 / 5(0)$ & 0.562 \\
Survival (\%) & & & & \\
$\quad$ 14-day survival (\%) & $5 / 5(100)$ & $3 / 5(60)$ & $4 / 5(80)$ & 0.287 \\
1-month survival (\%) & $4 / 5(80)$ & $3 / 5(60)$ & $3 / 5(60)$ & 0.741 \\
Operative complications (\%) & $2 / 5(40)$ & $2 / 5(40)$ & $0(0)$ & 0.256 \\
\hline
\end{tabular}

EOS: end of study.

Over the 30-day follow-up period no animals died in protocol or developed signs of tissue necrosis. No stenosis or occlusion were found at the carotid artery access site. In total, 5 of 15 animals required euthanasia before the end of the study (Table 3 ). In the control group, one was euthanized on day 13 due to severe diarrhea and another on day 16 due to coenurosis cerebralis. Two animals in the CS group and one animal in the BMS group developed systemic infection and were euthanized on post-injury day 3,12, and 18, respectively. At autopsy, the source of infection was identified as the site of tissue dissection around the left EIA (a zone of manipulation with the iliofemoral lymphatic node). Another animal in the BMS group developed an asymptomatic lymphocele in the zone of operation.

\section{DISCUSSION}

The current study is the first reported evaluation of bare metal and covered endovascular stents in a traumatic ovine model of peripheral blunt arterial injury resulting in occlusion. Both types of stent were demonstrated to 

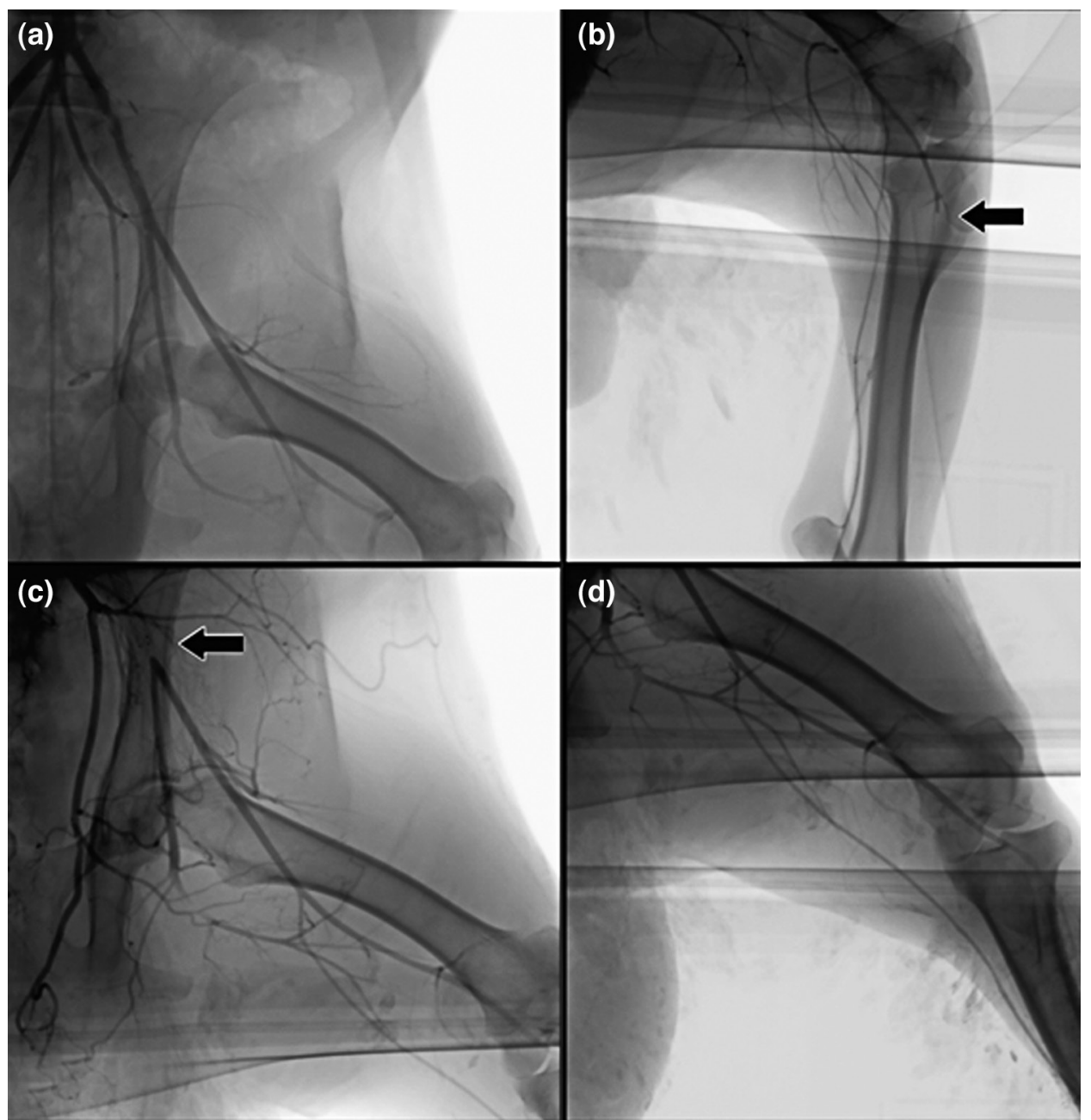

Figure 4 Representative completion angiograms of the injured hind limb 1 month after the injury and bare-metal stent implantation. $(\mathbf{a}, \mathbf{b})$ Angiograms demonstrate the only patent stent, with embolization of the distal part of the popliteal artery (arrow). (c, d) Angiograms show the occluded stent (arrow) of another animal with good collateral supply from the deep femoral artery and no distal emboli.

be effective in restoring initial arterial patency, but distal emboli and uniform in-stent thrombosis was seen beyond day 3 .

This work represents an extension of our group's efforts to further develop and refine an animal model of occlusive arterial injury [15]. We have previously described a model of blunt arterial injury that demonstrates the compatibility of the ovine arterial tree with endovascular interventions. The use of laparoscopy in the current model permits access to a more proximal portion of the animals' arterial tree, allowing for the generation of occlusion in a larger caliber artery. However, dissection in lymphatic nodes and vessels led to relatively high animal long-term morbidity. EIA thrombosis resulted in significant ischemic injury manifested by early lactate elevation and limb dysfunction in the control group. Lactate level increased in all groups on day 1 followed by sequential regress. The absence of

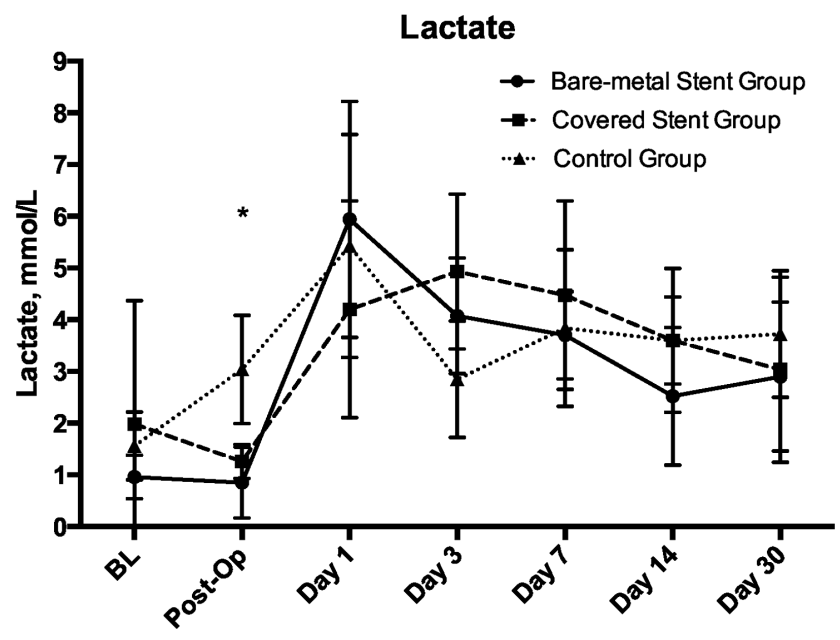

Figure 5 Time course changes in lactate level. An asterisk shows significant difference between groups. Data are presented as mean with standard deviation. BL: baseline. 
amputations and timely recovery of limb function can be explained by a sufficient collateral supply.

This research also adds to the limited body of laboratory and clinical evidence examining the role endoluminal stenting in traumatic vascular injury [13,14]. Tang and co-workers evaluated 2-month patency rate after CS deployment into a 2 -cm defect of the ovine SFA [13]. Nearly half of the stents were patent at the end of that study, but the animals were administered aspirin daily. Belczak and co-authors developed a porcine model of partial and complete carotid arterial transection to evaluate the feasibility of CS deployment [15]. The authors demonstrated that if a wire successfully passed through the injury zone, a CS can be easily deployed. However, arterial patency was only assessed at the time of implantation when the animal was fully heparinized. Using our previous ovine model [14], we were able to demonstrate the feasibility of BMS implantation into a zone of arterial traumatic occlusion [16]. All stented arteries remained patent during the 7-day follow-up period; however, the animals were fully heparinized during the study time frame, which is rarely feasible in a real trauma scenario.

The current study addresses the most challenging trauma scenario - limb ischemia with contraindication for post-operative systemic anticoagulation. However, it must be noted that intraoperative heparin was administered in this protocol. Modern imaging modalities (US, CTA, and DSA) allowed definitive evaluation of the injured artery for relatively long follow-up. BMS and CS deployment, the two main endovascular options for the treatment of peripheral arterial lesions, could be clearly investigated and compared in terms of arterial occlusion. The lesion was easily traversed by a wire and a delivery system, but it is likely that thrombus was not limited to the site of injury and embolized distally. This may have contributed to the reduction in distal flow and early in-stent occlusion. Although stent deployment in this model was feasible and straightforward, it cannot be viewed as a definitive revascularization, as demonstrated by a high rate of re-occlusion on day 3 .

The concept of temporary reperfusion is well established in the form of open temporary vascular shunting (O-TVS). A variety of conduits have been described, with successful shunt times beyond 24 hours reported, without anti-coagulation. The findings from the current study lead to the question - can an endoluminal stent perform as a method of endovascular temporary vascular shunt (E-TVS)? This is conceptually attractive for certain anatomical regions that are difficult to reach surgically, especially if a patient is undergoing another endovascular intervention. Rapid endovascular restoration of blood flow (endo-shunting) can theoretically allow focus to remain on other more critical injuries and permit simultaneous resuscitation. In trauma surgery, there is no single solution to a particular clinical problem and the utility of O-TVS or E-TVS will depend on many factors. Open shunting allows for back bleeding, embolectomy, and wound exploration. E-TVS permits rapid restoration of perfusion, depending on the morphology of the lesion, without the violation of the overlying tissue.

The concept of E-TVS been described in limited terms within the literature. In a recent paper, Rohlffs et al. reported a case in which a through-and-through wire technique followed by three CS deployments was successfully used as a temporary bridging tool for a completely transected axillary artery resulting from blunt trauma [9]. Some other techniques have recently been proposed to maintain limb perfusion $[17,18]$.

There are several limitations to this study. First, the distal thrombi were not quantified and no attempts were made to retrieve them. This was deliberate, as the intention was to evaluate a pragmatic endovascular procedure, accepting that small emboli were unlikely to significantly compromise limb function. In a clinical scenario, such a procedure will often result in peripheral smaller caliber artery occlusion and be ultimately compensated by collateralization. Second, although there was no postoperative anticoagulation, intraoperative anticoagulation was given. Our prior work and the published literature demonstrated that sheep have a tendency toward hypercoagulability $[19,20]$. Catheters and sheaths are easily thrombosed if heparin is not used at all. Because of the acute coagulopathy seen in trauma patients, this initial bolus of heparin may better represent the milieu in which one of these stents may be used. Although no strong contraindications exist for post-operative (post-stenting) anti-platelet therapy (APT) in trauma, we avoided it in this pilot study. Future research is warranted to evaluate long-term patency of E-TVS of traumatic occlusion in case of APT administration and/or associated brain injury. Our model focuses predominantly on grade IV level of blunt arterial injury and cannot be translated to evaluate lower grades of blunt injury resulting in intimal disruption, pseudoaneurysm, or dissection. Whilst clinical equipoise might exist in stenting lower grade, peripheral, arterial injuries, another model should be applied to study this.

Despite these limitations, the current study demonstrates that both BMS and CS deployment can restore perfusion acutely in an ovine model of thrombotic arterial occlusion. Without anticoagulation, however, thrombosis occurs by day 3 . Endovascular stenting may be an option for temporary restoration of flow as a damage control strategy.

\section{Ethics Statement}

(1) All the authors mentioned in the manuscript have agreed to authorship, read and approved the manuscript, and given consent for submission and subsequent publication of the manuscript.

(2) The authors declare that they have read and abided by the JEVTM statement of ethical standards including rules of informed consent and ethical committee approval as stated in the article. 


\section{Conflicts of Interest}

The authors declare that they have no conflicts of interest.

\section{Funding}

This study was funded by a grant from the RSF \#17-1801444 (2017).

\section{Author Contributions}

VAR and MJM carried out the study design, data interpretation, and manuscript writing. AVD, SYT, ABS, ISZ and VNA performed the data analysis and interpretation. IMS and KI carried out critical revision and work supervision.

\section{REFERENCES}

[1] Rozycki GS, Tremblay LN, Feliciano DV, McClelland WB. Blunt vascular trauma in the extremity: diagnosis, management, and outcome. J Trauma. 2003;55(5):814-24.

[2] Tan T-W, Joglar FL, Hamburg NM, et al. Limb outcome and mortality in lower and upper extremity arterial injury: a comparison using the National Trauma Data Bank. Vasc Endovascular Surg. 2011;45(7):592-7.

[3] Kauvar DS, Kraiss LW. Vascular trauma. In: Cronenwett JL, Johnston KW, editors. Rutherford's Vascular Surgery. 8th ed. London: Saunders Elsevier; 2014. pp. 2485-500.

[4] Scott AR, Gilani R, Tapia NM, Mattox KL, Wall MJ, Suliburk JW. Endovascular management of traumatic peripheral arterial injuries. J Surg Res. 2015;199(2):557-63.

[5] DuBose JJ, Savage SA, Fabian TC, et al; AAST PROOVIT Study Group. The American Association for the Surgery of Trauma PROspective Observational Vascular Injury Treatment (PROOVIT) registry: multicenter data on modern vascular injury diagnosis, management, and outcomes. J Trauma Acute Care Surg. 2015;78(2):215-22.

[6] Lönn L, Delle M, Karlström L, Risberg B. Should blunt arterial trauma to the extremities be treated with endovascular techniques? J Trauma. 2005;59(5):1224-7.

[7] Branco BC, DuBose JJ, Zhan LX, et al. Trends and outcomes of endovascular therapy in the management of civilian vascular injuries. J Vasc Surg. 2014;60(5): 1297-307.
[8] Zimmerman P, d'Audiffret A, Pillai L. Endovascular repair of blunt extremity arterial injury: case report. Vasc Endovascular Surg. 2009;43(2):211-4.

[9] Rohlffs F, Larena-Avellaneda AA, Petersen JP, Debus ES, Kölbel T. Through-and-through wire technique for endovascular damage control in traumatic proximal axillary artery transection. Vascular. 2015;23(1): 99-101.

[10] Joo JY, Ahn JY, Chung YS, et al. Therapeutic endovascular treatments for traumatic carotid artery injuries. J Trauma. 2005;58(6):1159-66.

[11] Hutto JD, Reed AB. Endovascular repair of an acute blunt popliteal artery injury. J Vasc Surg. 2007;45(1): 188-90.

[12] Stewart DK, Brown PM, Tinsley EA, Hope WW, Clancy TV. Use of stent grafts in lower extremity trauma. Ann Vasc Surg. 2011:25(2);264.e9-13.

[13] Tang AL, Diven C, Zangbar B, et al. The elimination of anastomosis in open trauma vascular reconstruction: A novel technique using an animal model. J Trauma Acute Care Surg. 2015:79(6);937-42.

[14] Reva VA, Morrison JJ, Denisov AV, et al. Development of an ovine model of occlusive arterial injury for the evaluation of endovascular interventions. Vascular. 2016; 24(5):501-9.

[15] Belczak S, Silva ES, Aun R, et al. Endovascular treatment of peripheral arterial injury with covered stents: an experimental study in pigs. Clinics (Sao Paulo). 2011;66(8): 1425-30.

[16] Reva VA, Morrison JJ, Denisov AV, et al. Bare metal stents can maintain arterial patency in traumatic occlusion. J Endovasc Resusc Trauma Manag. 2018;2(1):2-9.

[17] Davidson AJ, Neff LP, DuBose JJ, Sampson JB, Abbot CM, Williams TK. Direct-site endovascular repair (DSER): a novel approach to vascular trauma. J Trauma Acute Care Surg. 2016;81(5 Suppl 2):S138-43.

[18] Österberg K, Falkenberg M, Resch T. Endovascular technique for arterial shunting to prevent intraoperative ischemia. Eur J Vasc Endovasc Surg. 2014;48(2): 126-30.

[19] Blunt MH. Cellular elements of ovine blood. In: Blunt $\mathrm{MH}$, editor. The Blood of Sheep: Composition and Function. New York: Springer-Verlag; 1975. pp. 29-44.

[20] Byrom MJ, Bannon PG, White GH, Ng MK. Animal models for the assessment of novel vascular conduits. J Vasc Surg. 2010;52(1):176-95. 